\title{
SARS-CoV-2 Rapid Detection System using Electric Current-Spectrum for Human Exhaled Air Samples
}

\author{
Mohammad Bqoor ${ }^{1 \star}$, Maha Albqoor ${ }^{2}$
}

${ }^{1}$ Independent Researcher, Ph.D. Physics, Abu Dhabi, UNITED ARAB EMIRATES

${ }^{2}$ University of Jordan, Amman, JORDAN

*Corresponding Author: mohd.bqoor@gmail.com

Citation: Bqoor M, Albqoor M. SARS-CoV-2 Rapid Detection System using Electric Current-Spectrum for Human Exhaled Air Samples. Electron J Gen Med. 2021;18(1):em266. https://doi.org/10.29333/ejgm/8944

\section{ARTICLE INFO}

Received: 10 Apr. 2020

Accepted: 24 Aug. 2020

\begin{abstract}
The SARS-COV-2 Rapid Detection System is a SARS-COV-2 Electro-Spectroscopy detection system. The preliminary design of this system was studied theoretically in this paper. This system can detect the existence of sub-micro impurity particles in the human exhaled air, based on the unique shape, dimensions and density of these submicro impurities, such as viral particles, including CORONA viruses. This information is carried out by electron current buildup forming Electric Current-Spectrum (ECS) distinguishing the contents of the exhaled air. The design is based on Flashing Ratchet Potential (FRP) and beam of free electrons passing through the electrodes of the FRP. The ECS is characterized by curve deviations caused by interaction of low energy electrons' ( $\beta$ radiation) with matter, which depends on the matter's shape, dimension and density. This interaction causes a scattering (delaying) or absorption of the electrons by matter, by elastic or inelastic collisions, respectively. The ability of FRP to drift back the delayed electrons to their initial points can be used to characterize the produced ECS. The effect of delayed electrons can be amplified to form a visualized deviation in the ECS by electron multiplication system and can be interpreted into distinguishable SARS-COV-2 Barcodes or Fingerprints.
\end{abstract}

Keywords: SARS-COV-2 Rapid detection System, Electric Current-Spectroscopy, Flashing Ratchet Potential, electrons interaction with matter

\section{INTRODUCTION}

Coronavirus virions are semi-spherical objects with diameters of approximately $125 \mathrm{~nm}$ as depicted in recent studies by cryo-electron tomography and cryo-electron microscopy [1,2]. Hence, they are larger than other elements and compounds released by human coughs or exhalation. This special size and shape of Coronaviruses allow their detection by a new design of detector that is based on FRP. This FRP is operated in quantized frequency modes, and free electrons that travel as beams in accordance to the FRP electric and geometric specifications. This forms electric pulses that travel through the air sample medium in trajectories governed by air sample contents.

\section{DESCRIPTION OF THE SYSTEM}

The FRP is an arrangement of positive and negative electrodes fixed in a line as shown in Figure 1. The electrodes can be a mesh (grid) of metal and insulated by a coating layer of dielectric material. This sequence of electrodes is laid inside an insulating tube. The electrodes charge and the spatial arrangement are described as:

- The voltage of the electrodes is arranged in sequence setup as " $+\mathrm{V},-\mathrm{V},+\mathrm{V},-\mathrm{V}, \ldots$..;
- The electrodes charged will be varying frequently; the charge equals $(0)$ during voltage cut-off time period $(\tau)$, and equals $(Q)$ during the voltage On phase time period $\left(\tau^{\prime}\right)$; and

- Electrodes are laid in parallel, with spacing $(d)$ between electrodes with opposite charge signs, and spacing $(D+d)$ between electrodes with similar charge signs as shown in Figure 1.

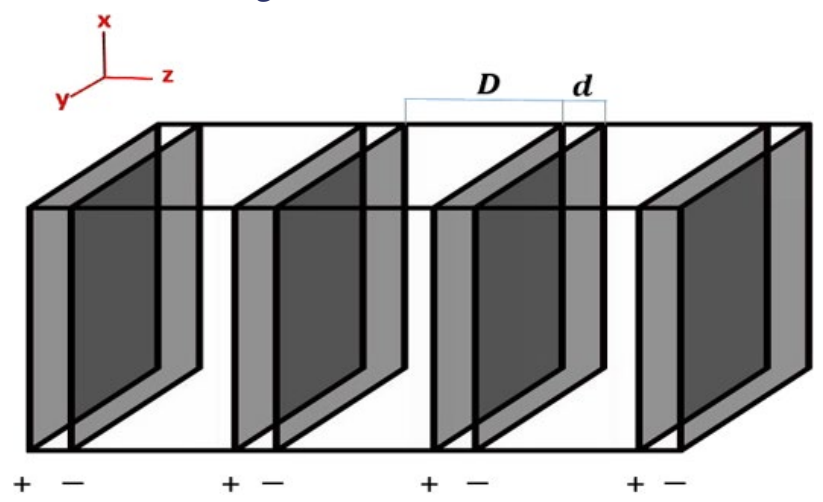

Figure 1. Arrangement of Electrodes

The shape of the produced electric potential is displayed in Figure 2. The resulting electric potential has the shape of ratchet potential or Sawtooth potential [3], and has local extreme values (Wells and Saddles). The geometry and electric specifications of the FRP are described in the next sections. 


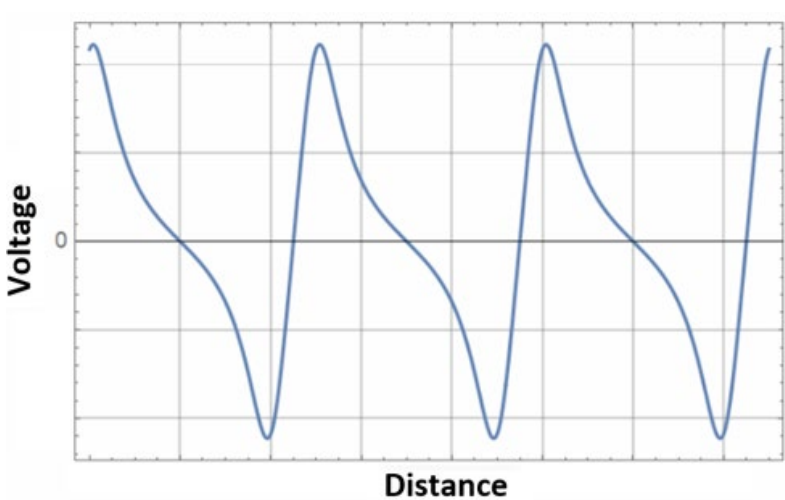

Figure 2. Electric Potential of the Electrodes

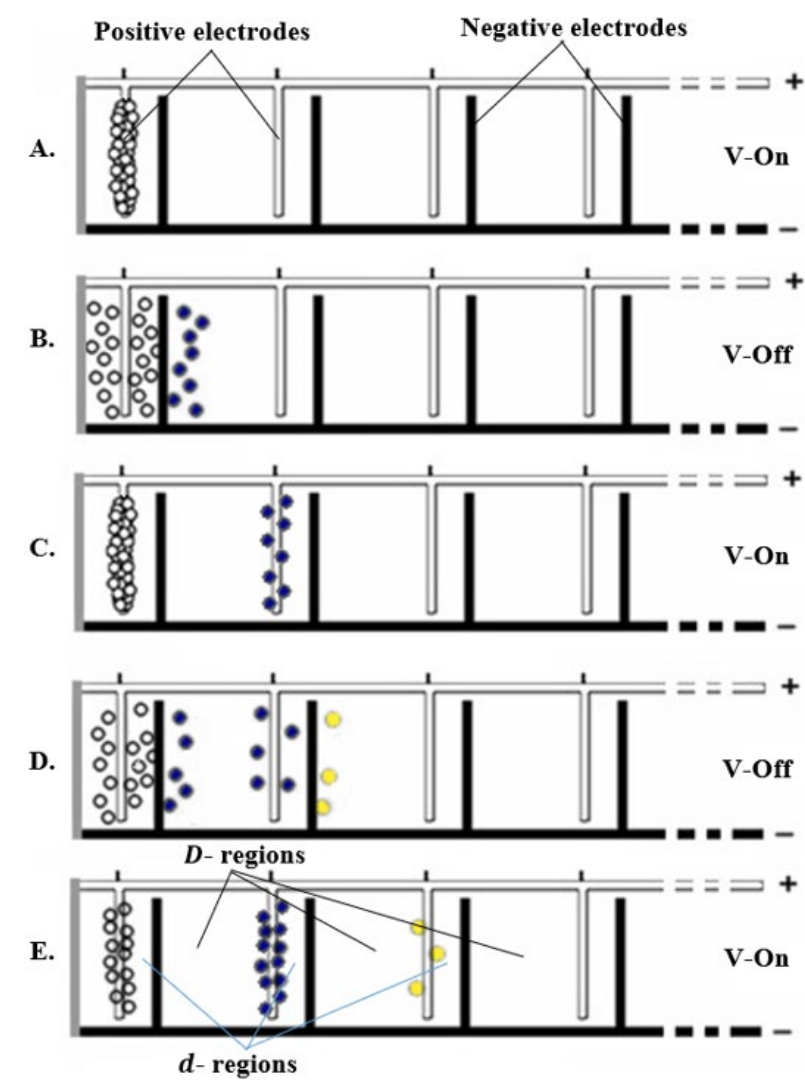

Figure 3. Ratcheting Mechanism

\section{WORKING METHODOLOGY}

The working methodology and free electrons behavior in the FRP are described in the "Ratcheting Mechanism" described as the following:

1. In the initial stage, the voltage is applied and the free electrons are mounted in the region of the first (left) positive electrode shown in Figure 3A.

2. When cutting the voltage for a while $(\tau)$ as shown in Figure 3B, electrons start moving with equal speeds (equal applied voltage) inside the system and crossing the $d$ - region, then $D$ - region and so on. The traveled distance depends on the following factors:

a) The cut-off period $(\tau)$, where the traveled distance increases by increasing $(\tau)$; b) The speed of electrons $(v)$, which depends on the voltage of the FRP electrodes, where the traveled distance increases by increasing $(v)$;

c) Medium electrical resistivity, which depends on the medium contents (particles individual shapes, dimension, and density);

d) The location of the free electron's starting point; and

e) The electromagnetic forces in the medium.

3. At the end of the cut-off period $(\tau)$, and applying the voltage on the electrodes for a period $\left(\tau^{\prime}\right)$, the electrons failed to reach the first negative electrode, i.e. remain in the first $(d)$ region (White Circles), these electrons are drifted back by the electric field of the electrodes to the starting point (first positive electrode) as shown in Figure $\mathbf{3 C}$. The electrons succeed passing the first negative electrode (Blue Circles) and reach first $D$ region are drifted forward to the second positive electrode. These actions continue for the next cut-off periods, until the whole electrons reach the last positive electrode in sequence (pulse by pulse) as shown in Figure 3D and 3E.

4. The increase in the cut-off period (V-Off) increases the number of electrons that are allowed to reach the next negative electrodes and pass to the next $D$ - regions.

5. The pulse buildup forms distinct Electric CurrentSpectrums (ECSs) as shown in the example in Figure 4. The three smooth ECSs belong to a pure air sample, and the other three ECSs refer to infected (viral contents) air sample for different cut-off periods $\left(\tau_{1}, \tau_{2}, \tau_{3}\right)$.

6. The general trend of the ECS is a sigmoid function having a characteristic " $\mathrm{S}$ "-shaped curve, because of the ratcheting mechanism (explained in Figure 3 ) and the limited number of electrons.

7. The buildup of electrons reaching the last positive electrode in the pure sample is faster than in the infected sample. Because of that number of free electrons scattered by the viral contents, these electrons fail to pass the negative electrode and then drifted back to their starting points once the cut-off period ends. In addition, the free electrons trajectories lengths are increased by increasing the content of matter surrounding these trajectories, depending on matter's specifications (shape, dimension, and density).

8. The effect of the variation in the cut-off period on the ECS is illustrated in Figure 5; by considering the lengths of electron trajectories governed by the shape, dimension, and density of the objects laid in these trajectories. The periods $\left(\tau_{1}, \tau_{2}, \tau_{3}\right)$ determine which trajectories allow their electrons to pass the $d$ - region as shown in the dashed-lines in Figure 5 . The deviations in the infected sample's ECS are representing viral particles. Theses deviation can produce a distinguishable Barcodes or Fingerprints of specific infected sample.

9. The deviations in the infected sample's ECS are representing viral particles. Theses deviation can produce a distinguishable Barcodes or Fingerprints of specific infected sample. 


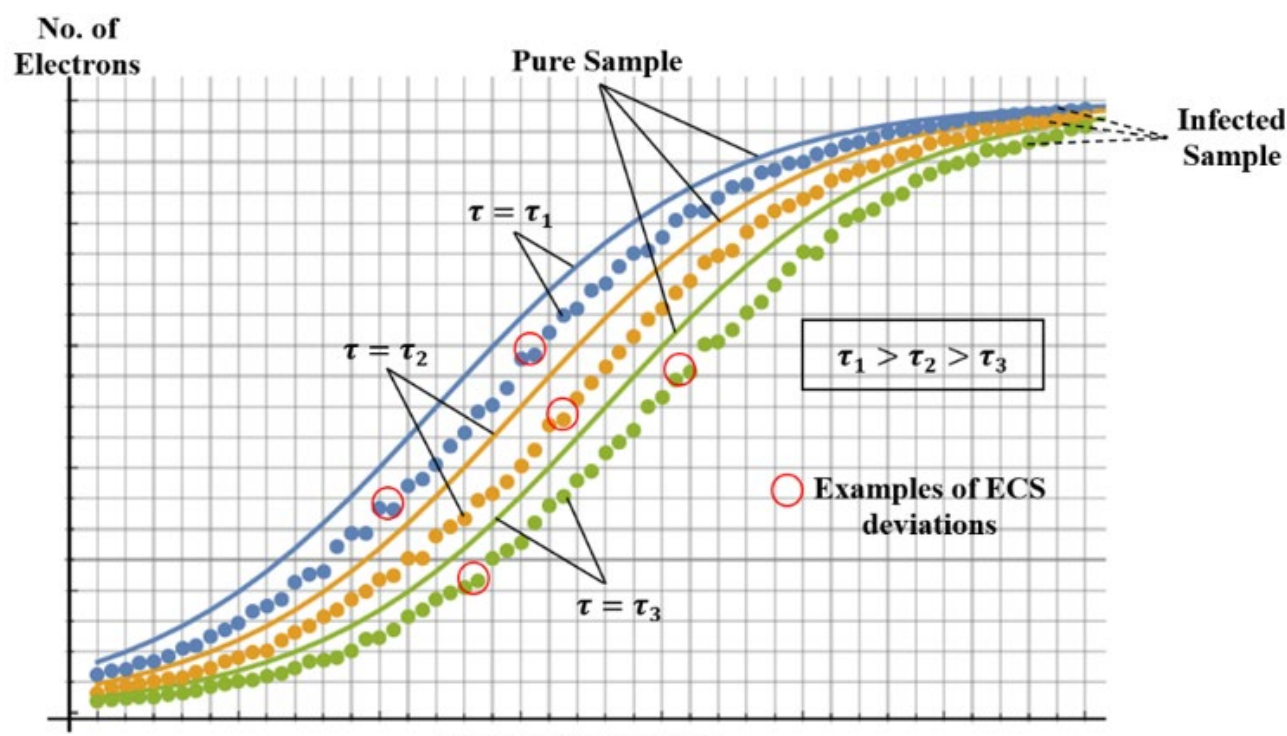

No. of cut-off periods

Figure 4. Electric Current-Spectrums

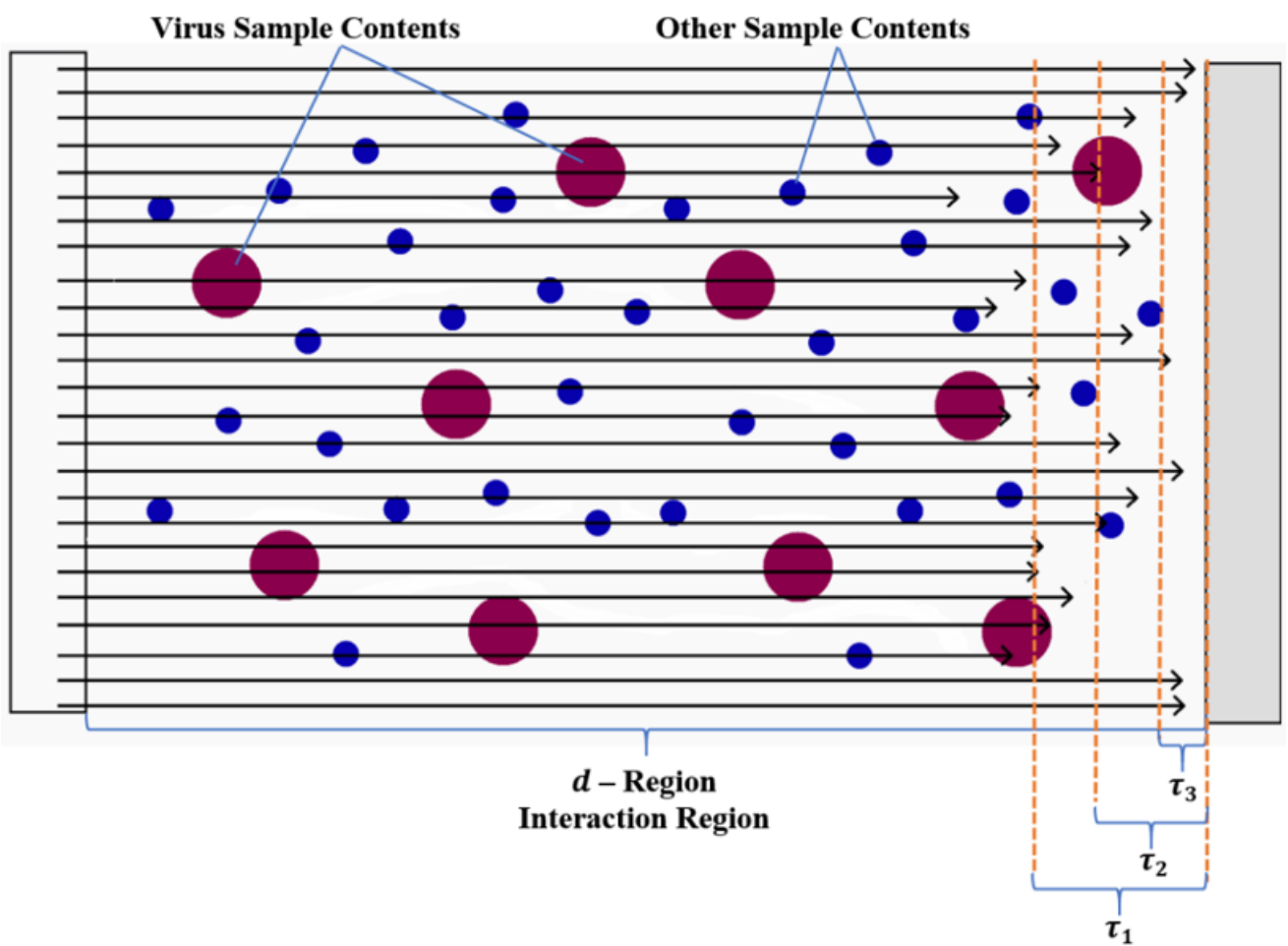

Figure 5. Electrons Trajectories in the $d$ - Region During Cut-Off Time Period

10. The variation in the cut-off period can be used to expand or visualize the ECS deviations, as well as the FRP voltage and geometry. The variations in the cut-off period shall be in practically developed quantized operation frequencies "Operational Log" to handle the specific shape, dimension, and density of the viral particles. This can be done based on practical tests on both positive and negative SARS-COV-2 on a wide range (significant statistical number of samples), taking into consideration disease stage and other human physical characteristics, such as age, weight, temperature, wellness, etc. In addition, several Barcodes or Fingerprints can be developed for different types of viral particles by making a database based on practicing this system.
11. The largest deviations in the ECS belong to the largest and most dense objects in the sample, while the small increments disappear in case of coincidence with large increments, as known in the spectroscopy analysis as Coincidence Summing Phenomena. Therefore, filters shall be added to ensure that the viruses are the majority of large objects in the sample. Also, the air sample should not include liquid droplets or other objects larger than viruses. This can be effectively achieved by using a microfilter to exclude objects larger than ( 0.5 micrometer), while the impurities in liquid droplets (including potential virus particles) can be extracted from droplets by several means, such as forced diffusion or closed air sample circulation, in which droplet low temperature evaporation occurs, 


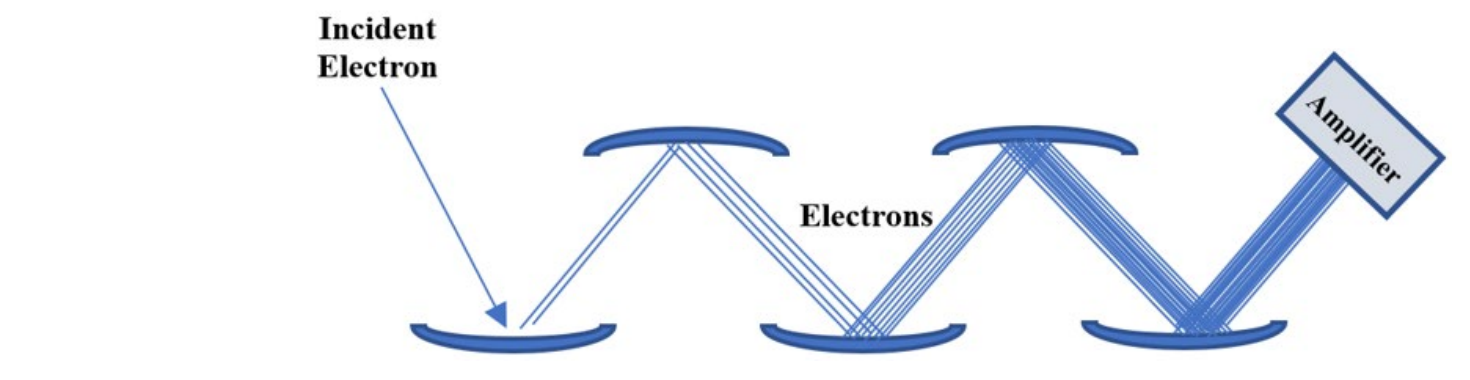

Figure 6. Electron Multiplying Tube

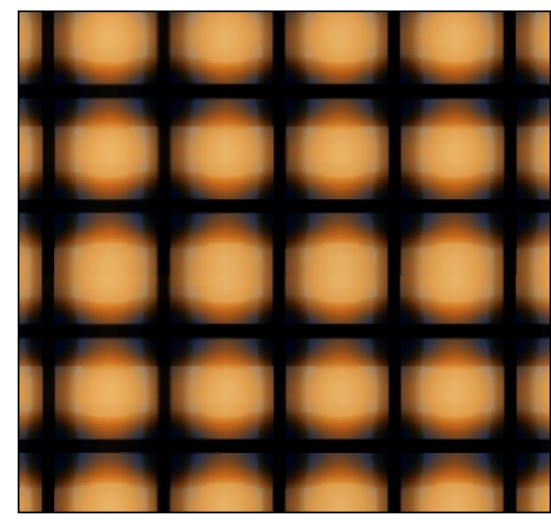

Figure 7. Electric Potential of the Electrodes

and low pressure can accelerate such process. Moreover, to increase the recognition efficiency, an additional filter can be added to remove particles that are slightly smaller than viruses' dimensions (less than $\sim 0.05$ micrometer) filter.

12. The sample preparation explained in point 11 , will ensure that the majority of impurities in the exhaled air will be the objects that are in the viral dimensions. Then, the presence of ECS deviations will reflect the existence of objects in the viral dimensions, while the length of ECS deviations will recognize the viral types. Moreover, the frequency of ECS deviations will define the density of viral particles in the sample.

13. Electron pulses that reach the last positive electrode of the FRP can be detected and amplified by using electron-multiplying tube [4] as shown in Figure 6.

\section{The Distribution of Free Electrons in the System}

There are two electric forces govern the distribution of free electrons in the system; the first one is the electric forces between electrodes and free electrons, and the second one is the mutual electric forces between free electrons themselves. Hence, the factors determining the distribution of free electrons in the system are:

- The voltage of the electrodes;

- The number of free electrons; and

- The geometry of the electrodes.

According to these two forces, and for mesh shape electrodes, the free electron charge density takes the pattern presented in Figure 7. The figure shows that the darker the color has more density of free electrons.

All free electrons are laid in equipotential locations [5] to comply with the static condition at absolute zero temperature, $(T=0 \mathrm{~K})$, otherwise, if there is an electric potential difference between any two locations, electric force will be non-zero. Therefore, the free electron moves toward the higher electric potential locations and violates the static condition.

\section{Electron Mean Free Path in Air Samples}

Because of electromagnetic (EM) forces on the electrons, as well as, the ionization interactions (Electron-Matter) the mean free path of the beam of electrons ( $-\beta$ particles) in the air is quite complicated. These effects can be minimized by a suitable adjustment of the cut-off period that will ensure such scattered particles not reaching the $D$-region. In addition, making balance among the electron speed (electrodes voltage), the EM effect (decreased by increasing the voltage) and the ionization effect (increased by increasing the voltage) can minimize the EM and ionizing effects. Moreover, the electron is replaced by a heavier ion (such as protons $\mathrm{H}^{+}$) that moves in more linear trajectories and inverting the electrodes charge signs. This helps handle the change of the charge.

The mean free path of the beam of electrons ( $\beta$ particles) in the air can be interpreted in the concept of Mass Attenuation Factor [6]. This factor depends on the energy of electrons and the medium passing through. The voltage, the number of free electrons and the geometry (mainly the length of the $d$ - region) of the FRP shall be manipulated to allow a significant number of free electrons to pass the $d$-region successfully and then to be drifted forward to the next positive electrode. The rest of the free electrons that experienced one or more collisions (elastic or inelastic collisions) with the air samples' contents are drifted back to the starting point. The majority of electrically polarized air sample objects are filtered, then the electromagnetic force (Lorentz Force) becomes effective only in very short distances (in the order of molecular distances) between free electrons and sample molecules.

\section{Free Electrons Interaction with Sample Particles}

The sample content particles have random locations and random velocities in accordance with Boltzmann Distribution. $O$ the other hand, the air sample has net drift speed normal to the FRP axis (samples passage) as shown in Figure 8. However, mixing the air sample (common viral particle per volume passing the $d$-region), makes the superposition (or shadowing) viral particles negligible, taking into consideration the following ratio:

$$
\frac{\text { viral individual volume }}{\text { rational } d-\text { region volume }} \sim 10^{-13}
$$

The range of viral contents in air samples is $10^{3}-10^{6}$ particles/Liter [7]. Then the random distribution does not significantly affect the current characteristics. 


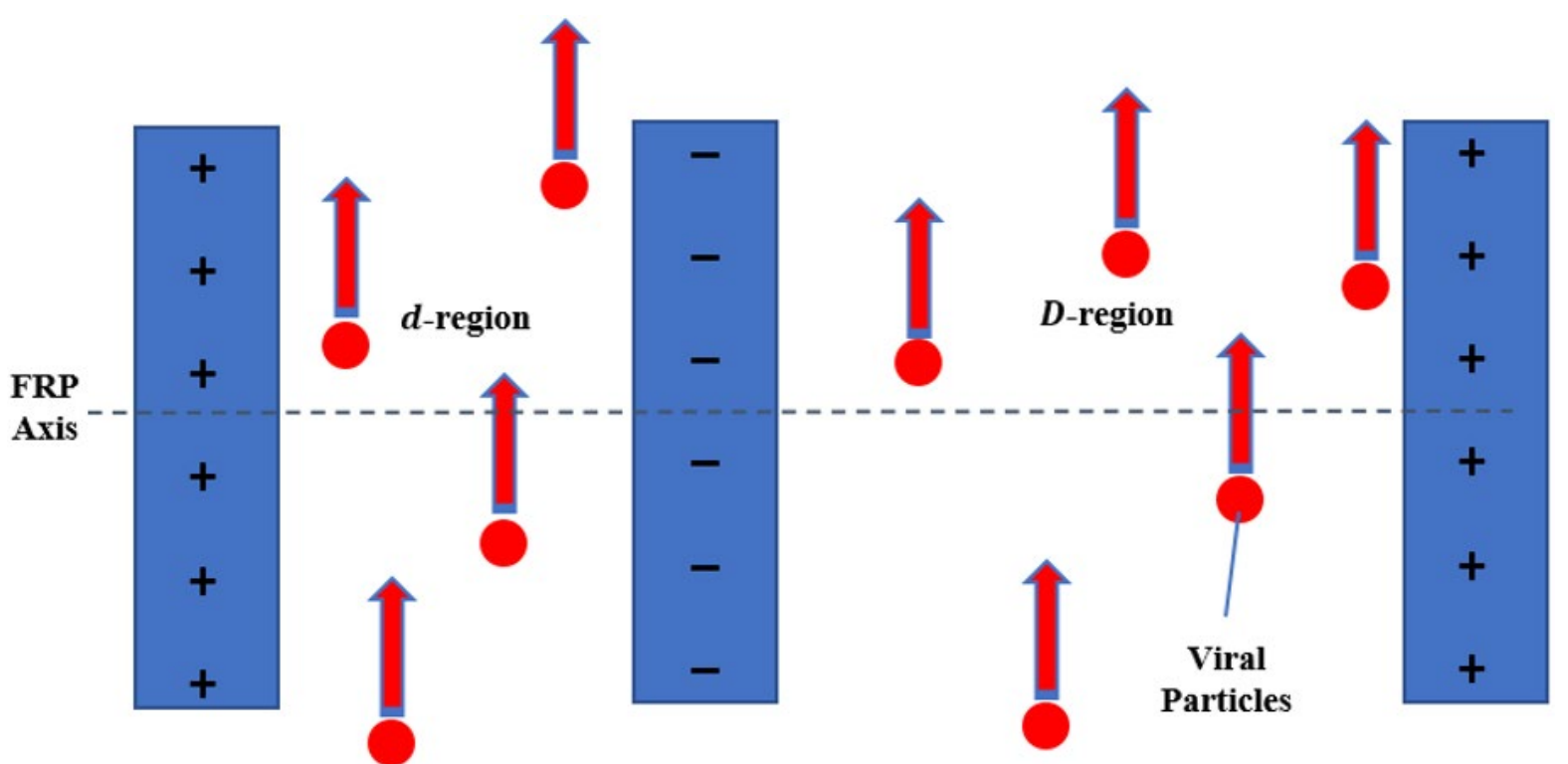

Figure 8. Samples passage through FRP
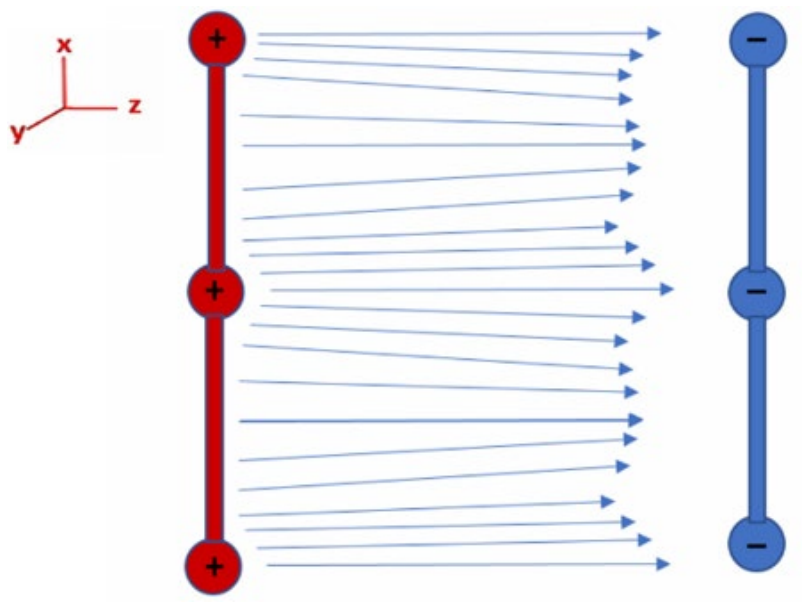

Figure 9. $(x-y)$ plane pulse-free electrons

The free electrons are released; directly after electrode voltage cut-off, from different initial locations (adjacent to the first positive electrode). These initial locations have slightly different lengths from the end of $d$-region as shown in Figure 9. Considering that mutual electron-electron repulsion forces ensure that the majority of trajectories moving in parallel to the $z$-axis as displayed in Figure 9. Hence, producing semi $(x-y)$ plane pulse of electrons moving toward $z$-axis. These lengths differences appear in the background ECS (pure air sample), and their effect can be excluded by the subtraction (normalization) of the sample ECS from the background ECS. However, the smaller mesh gaps and thickness are the more accurate produced ECS is.

\section{OPERATION PARAMETERS AND OTHER CONSIDERATIONS}

1. The cut-off periods $\left(\tau_{1}, \tau_{2}, \tau_{3}, \ldots . ., \tau_{N}\right)$ are considered the main parameter of operating the system and forms the System Operational Log. It can be used practically to develop a database of SARS-COV-2 Barcodes or Fingerprints, corresponding to disease stage and other human physical parameters. The Algorithm of
Table 1. System's controllers, variables and unknowns

\begin{tabular}{|c|c|c|}
\hline Controllers & Variables & Unknowns \\
\hline $\begin{array}{l}\text { 1.Cut-off periods }(\tau) \text { : } \\
\text { Controlling ECS Slope and } \\
\text { Deviations. }\end{array}$ & 1. ECS Slope. & $\begin{array}{l}\text { 1.Density of Viral } \\
\text { Particles }\left(N_{D}\right) \text {. }\end{array}$ \\
\hline $\begin{array}{l}\text { 2.Free electron current } \\
\text { density, correlated with } \\
\text { system geometry and } \\
\text { Electrodes Voltage: } \\
\text { Controlling detection } \\
\text { accuracy and visualization. } \\
\text { 3.Mixing Air Sample with } \\
\text { pure gas: } \\
\text { Controlling relative Density } \\
\text { of Viral Particles }\left(N_{D}\right) \text {. }\end{array}$ & $\begin{array}{l}\text { 2. ECS Deviations } \\
\text { Dimensions } \\
\text { (relative to ECS } \\
\text { Slope). }\end{array}$ & $\begin{array}{l}\text { 2.Shape, dimension } \\
\text { and density of } \\
\text { Sample Content } \\
\text { Particles. }\end{array}$ \\
\hline
\end{tabular}

developing the Barcodes or Fingerprints governs the accuracy of this system.

2. The System Operational Log can be developed based on the (controllers, variables and unknowns) relations, as described in Table 1.

3. The voltage On phase period $\left(\tau^{\prime}\right)$ should be longer than the cut-off periods $(\tau)$ to allow electrons to be drifted toward the positive electrodes (across the $D$ - region), and to be arranged and mounted stationary (approximately) in the vicinity of positive electrodes.

4. The FRP dimensions and magnitude of electrodes voltages should be determined experimentally, however; the following constraints need to be taken into consideration:

a. The dimensions $(D, d)$ need to be optimized based on the manufacturing accuracy and limits (insulation/dielectric coating) and the breakdown voltage (distance between electrodes).

b. The numbers of electrons and electrodes voltage are correlated (the increase is the voltage; the increase number of electrons is). This is required to optimize the balance between the need for significant current density [high accuracy (ECS resolution)] and the $(D, d)$ manufacturing accuracy and limits. 


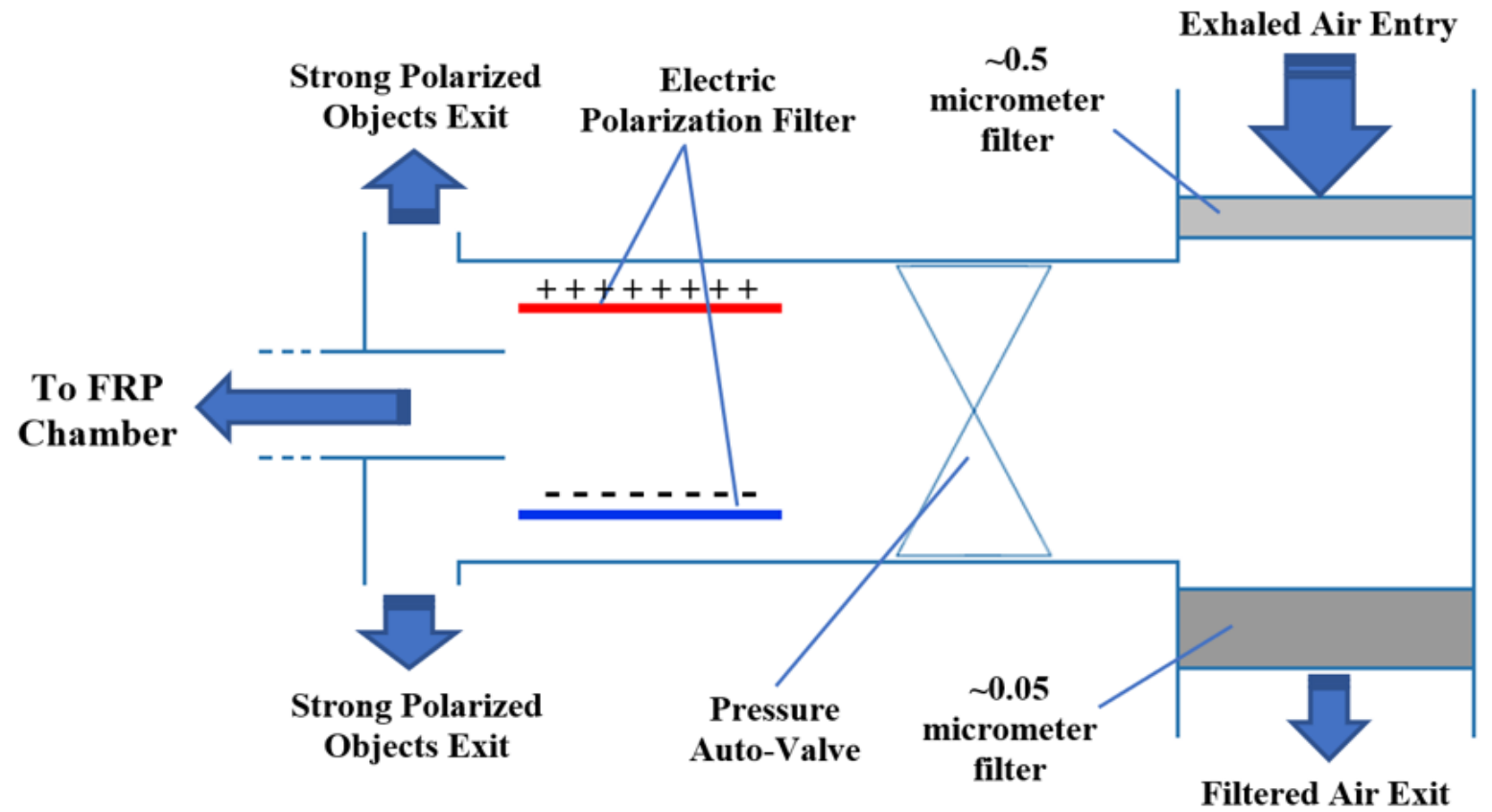

Figure 10. Sample Concentration Tube

5. Water vapor and other strong electric polarized objects in the sample content shall be excluded using an electric field (polarity filters) before entering the FRP region. This minimizes the electric distortion in the FRP caused by this type of particle. The structure of viruses consists of dielectric protein polymers [8] with a weak polarization compared to water.

6. The arrangement of the system filters (Large Objects Filter, Small Objects Filter, and Electric Polarization Filter) can be arranged as shown in Figure 10.

\section{RESULTS AND DISCUSSION}

The SARS-COV-2 Rapid Detection System was studied theoretically and shows promising functionality in prompt detection of viral particles in human exhaled air samples, including SARS-CoV-2 virus, taking into consideration the followings:

1. There are rare viruses that have almost similar size to COVID19, such as Herpes virus (125 nm), whereas viruses are different in their physical structure and chemical composition, despite that some viruses have similar size. Nevertheless, difference in the detailed shape and density (material and geometry) will give slightly different ECS deviations (i.e. different number of scattered and delayed electrons caused by different material and geometry) that might be distinguishable based on the system manufacturing precision and Operational Log. However, the existence of such viruses could give False Positive results and reduces the overall accuracy of the detection system, but the system still provide competitive test option by means of time and accuracy compared to other available SARS-COV-2 tests.

2. The best status of sample situation (stationary or circulation, periodicity of circulation, etc.) inside the system should be analyzed experimentally, taking into consideration the system ability to detect (recognize) the passage of virus in the $d$ - region. This method of operation reduces the operational time, which is needed in case of operation in crowded places (such as airports). In addition, circulation ensures minimizing the gravity effects on the viruses in the sample.

3. The system will detect only the infectious cases that have SARS-CoV-2 viral particles in their exhaled air.

4. This system can be utilized for other purposes such as air quality and other medical, industrial, and environmental applications.

\section{CONCLUSIONS}

The accurate and timely detection of SARS-COV-2 are one of the main challenges in facing the COVID-19 pandemic, were most of the available test consume time and resources. Both delayed and inaccurate results contribute in spreading the pandemic. Merging two physical concepts; Flashing Ratchet Potential and Radiation Interaction with matter, will produce an Electric Current Spectrum that contain information on the existence of sub-micro particles, that can be identified by the deviations in the Electric Current Spectrum curve.

Author contributions: All authors have sufficiently contributed to the study, and agreed with the results and conclusions.

Funding: No funding source is reported for this study.

Declaration of interest: No conflict of interest is declared by authors.

\section{REFERENCES}

1. Barcena M, Oostergetel GT, Bartelink W, Faas FG, Verkleij A, Rottier PJ, Koster AJ, Bosch BJ. Cryo-electron tomography of mouse hepatitis virus: Insights into the structure of the coronavirion. Proceedings of the National Academy of Sciences of the United States of America, 2009;106(2):582-7. https://doi.org/10.1073/pnas.0805270106 PMid:19124777 PMCid:PMC2613939 
2. Bibi F, Villain M, Guillaume C, Sorli B, Gontard N. A Review: Origins of the Dielectric Properties of Proteins and Potential Development as Bio-Sensors. Sensors (Basel), 2016;16(8):1232. https://doi.org/10.3390/s16081232 PMid:27527179 PMCid:PMC5017397

3. Bqoor M. Ionized Gas Thermoelectric Generator. Thermal Science and Engineering Progress. Volume 18, 1 August 2020, 100496. https://doi.org/10.1016/j.tsep.2020.100496

4. Giordano N. College Physics: Reasoning and Relationships. Cengage Learning. 2nd edition, 2015.

5. Mahajan C, Bagdia Arts RG, Lakhotia Commerce SB, Bezonji R. Mass attenuation coefficients of beta particles in elements. Science Research Reporter 2(2):135-141, 2012.

6. Mellon F. Mass Spectrometry, Principles and Instrumentation. in Encyclopedia of Food Sciences and Nutrition (Second Edition), 2003. https://doi.org/10.1016/ B0-12-227055-X/00746-X
7. Neuman BW, Adair BD, Yoshioka C, Quispe JD, Orca G, Kuhn $P$, Milligan RA, Yeager M, Buchmeier MJ. Supramolecular architecture of severe acute respiratory syndrome coronavirus revealed by electron cryomicroscopy. Journal of virology. 2006;80(16):7918-28. https://doi.org/10.1128/ JVI.00645-06 PMid:16873249 PMCid:PMC1563832

8. Nikitin N, Petrova E, Trifonova E, Karpova O. Influenza Virus Aerosols in the Air and Their Infectiousness. Hindawi Publishing Corporation Advances in Virology, 2014;859090. https://doi.org/10.1155/2014/859090

PMid:25197278 PMCid:PMC4147198 